\title{
Are physicians aware enough of patient radiation protection? Results from a survey among physicians of Pavia District- Italy
}

\author{
Francesca Campanella ${ }^{1{ }^{* \dagger}} \mathbb{D}$, Laura Rossi ${ }^{1 \dagger}$, Elio Giroletti ${ }^{2,3}$, Piero Micheletti ${ }^{1}$, Fabio Buzzi ${ }^{1}$ and Simona Villani ${ }^{1}$
}

\begin{abstract}
Background: Radiological practices are the first anthropic sources of ionizing radiation exposure of the population. However, a review of recent publications underlines inadequate doctors' knowledge about doses imparted in medical practices and about patient protection that might explain unnecessary radiological prescriptions. We investigated the knowledge of the physicians of Pavia District (Italy) on the risk of radiation exposure.

Methods: A cross sectional study was performed involving the Medical Association of Pavia District. Data were collected with a self-administered questionnaire, available on-line with private login and password.

Results: Four hundred nineteen physicians fulfilled the questionnaire; $48 \%$ of participants reported training about radiation protection. The average percentage of correct answers on the knowledge on ionizing radiation was 62.29\%, with a significantly higher result between radiologist. Around 5 and $13 \%$ of the responders do not know that, respectively, ultrasonography and magnetic resonance do not expose patients to ionizing radiations. Only $5 \%$ of the physicians properly identified the cancer risk rate associated to abdomen computed tomography.

Conclusions: The findings show a quite good level of the general knowledge about ionizing radiations, higher that reported in literature. Nevertheless, we believe the usefulness of training on the risk linked to radiation exposure in medicine for physicians employed in every area.
\end{abstract}

Keywords: Ionizing radiation, Radiation protection knowledge, Radiological procedures, Physicians' awareness, Radiation dose

\section{Background}

In accordance with the estimation of the Italian Agency for the Environmental Protection [1], the effective dose of ionizing radiation from medical sources is about $1.2 \mathrm{mSv} /$ year, while the amount of ionizing radiation from other men made sources is $0.0072 \mathrm{mSv} /$ year. Therefore medical exposure is the first anthropic source of ionizing radiation of the population.

In the last years, the annual number of radiological procedures is exponentially increasing, consequently the collective dose is growing up, especially because of the spread of medical practices imparting non-trivial doses to patients, such as diagnostic Computed Tomography (CT) and

\footnotetext{
* Correspondence: francesca.campanella@live.it

${ }^{\dagger}$ Equal contributors

'Department of Public Health, Experimental and Forensic Medicine,

University of Pavia, Via Forlanini 2, 27100 Pavia, Italy

Full list of author information is available at the end of the article
}

interventional radiology. This implies that the risk associated to ionizing radiation, although small at the individual level, might lead to an increased number of cancer cases in the population. The study of Berrington de Gonzales [2] estimated that CT scans performed during 2007 in the USA could be related to about 29,000 future cancers.

Public health policies should support radiation protection culture in medical practice in order to ensure safety cares and the best allocation of available resources [3].

Medical practices involving ionizing radiation are allowed only if justified [4]. The knowledge level on radiation safety of physicians represents a key point to reduce the patient's exposure in medical practices. Doctors have to know the doses, the detrimental effects associated and the alternatives with lower risk of exposure to avoid the request of unnecessary and unsuitable radiological exams. 
Nevertheless, several studies detected lack or poor knowledge about ionizing radiation doses and effects in radiological procedures by the doctors in every specialities and grades.

The aim of the study was therefore to investigate the physicians' knowledge about patient exposure to ionizing radiation during common radiological procedures and radiation protection awareness.

\section{Method}

\section{Design and population}

All physicians belonging to the Order of Physicians, Surgeons and Dentists of the Pavia District were eligible for the study. Dentists were excluded. As our survey was performed on the entire population of physicians of Pavia District (4410 individuals), is not expected to estimate the sample size.

A cross-sectional design was applied to achieve study aims.

The Ethics Committee of the University of Pavia approved the study protocol.

\section{Study plan and conduction}

The study was carried out from March to May 2013. The board member were invited to attend the study with a letter written by the Order's Chairman. Furthermore, an advertisement was included on the website of the Medical Association. Precisely, after 1-month from the first invitation, a reminder was sent to the physicians to improve the response rate. To prevent study bias from participant selfeducation, the actual topic was not clearly mentioned.

At each participant was asked to complete a semistructured questionnaire of 26 multiple-choice questions concerning radioprotection importance awareness, knowledge about radiation imaging doses and deterministic/ stochastic effects, education programs and demographical data (see Additional file 1). Correct answers are based on data published in the Annual Report of the Italian Agency for the Environmental Protection [1], Council Directive 2013/59/EURATOM [5], and in Hall EJ [6] and Mettler FA [7] papers.

The questionnaire was self-administered, anonymous and available by personal login and password on a specific web platform. The web database of the University of Pavia automatically captured survey responses. Each question had to be filled in to allow the database admitting the questionnaire. To avoid duplicate entries, the platform was able to prevent a second entry once the user completed the survey.

\section{Endpoints}

The level of knowledge on the topic of ionizing radiation is the primary end-point and was measured as the percentage of correct answers to questions of the knowledge section.

The secondary end points were the perception of physicians about the importance of knowledge in radiation protection, the attendance to previous specific training and the availability of updating in radiation protection. All these were measured by single items present in the questionnaire or combination of them.

\section{Statistical analyses}

Quantitative variables were summarized as means and standard deviation; categorical variables were processed as percentages. The 'percentage of correct answers' was computed summarizing the positive answers to the items about the knowledge and comparing to the total of the possible correct answers.

Differences in knowledge between specialist radiologists and the other specialists were investigated. For the quantitative variables unpaired t test with Satterthwaite's correction for unequal variances if necessary was applied. For qualitative variables chi-square test or Fisher's exact test as appropriate was used.

The statistical analyses were performed using STATA 12. A $p$ value $\leq 0.05$ was considered significant.

\section{Results}

Four hundred nineteen physicians participated to the study with a response rate of $10.5 \%$. These were from doctors specialised in the following macro-areas: general practice (114), specialized medicine (55), neurosciences (29), general surgery (18), specialized surgery (50), diagnostic and laboratory medicine (39), clinical services (41), public health (32). Forty-one doctors were not specialized in any area. The responders included $11.45 \%$ family physicians, $64.20 \%$ consultants, $19.33 \%$ resident physicians, $1.90 \%$ academics and $1.67 \%$ chief medical officers; in two questionnaires, the occupation was not indicated. The mean age was 44.76 years $( \pm 12.33)$ and around half of participants $(59.43 \%)$ had been practicing for more than 10 years. Demographic variables of responders are illustrated in Table 1.

Only 202 (48.2\%) responders attended formal radiation protection courses during Academic period or professional career. When education was received, it was more commonly in the form of Academic course (76.14\%) or Continuing Medical Education (CME) programs (42.62\%). $22.91 \%$ of doctors indicated to be interested in updating courses on radioprotection. Three hundred sixty-four doctors ordered or performed diagnostic imaging test, mostly in accordance with personal choice $(65.9 \%)$ or with the specialist dispositions $(25.9 \%)$. Almost all of this physicians considered radiation protection topics 'very important' or 'quite important'. On the contrary, only $69,45 \%$ of all responders believed it. 
Table 1 Demographic variables of responders

\begin{tabular}{|c|c|c|}
\hline & Frequency & Percentage \\
\hline \multicolumn{3}{|l|}{ Gender } \\
\hline Male & 210 & 50.12 \\
\hline Female & 209 & 49.88 \\
\hline \multicolumn{3}{|l|}{ Years of experience after graduation } \\
\hline$<10$ years & 170 & 40.57 \\
\hline$>10$ years & 249 & 59.43 \\
\hline \multicolumn{3}{|l|}{ Macro-areas of Specialty } \\
\hline General practice & 114 & 27.21 \\
\hline Specialized medicine & 55 & 13.13 \\
\hline Neurosciences & 29 & 6.92 \\
\hline General surgery & 18 & 4.30 \\
\hline Specialized surgery & 50 & 11.93 \\
\hline Diagnostic and laboratory medicine & 39 & 9.31 \\
\hline Clinical services & 41 & 9.79 \\
\hline Public health & 32 & 7.64 \\
\hline None & 41 & 9.79 \\
\hline \multicolumn{3}{|l|}{ Occupation } \\
\hline Family physician/paediatrician & 48 & 11.45 \\
\hline Consultant & 269 & 64.20 \\
\hline Chief medical officer & 7 & 1.67 \\
\hline Resident & 81 & 19.33 \\
\hline Academic & 8 & 1.90 \\
\hline Other & 6 & 1.43 \\
\hline Mean age & \multicolumn{2}{|c|}{44.75 years \pm 12.33} \\
\hline
\end{tabular}

In average the proportion of correct answers to the questions on ionizing radiation knowledge was $62.29 \%$, slightly greater for the senior doctors with more than 10 years of job experience than for junior doctors with less than 10 years of job experience $(63.97 \%$ vs $61.14 \%$; $t=2.49, p=0.01)$. The proportion was significantly higher also between radiologists $(73.95 \%)$ compared to other specialist $(61.32 \%)(t=5.91, p<0.0001)$. No difference were found between doctors attending specific training and those not attending. Overall, $88.07 \%$ of participants answered correctly at almost the half of knowledge questions.

The most part of physicians rightly indicated that Ultrasound (US) and Magnetic Resonance (MRI) don't imply use of ionizing radiation $(94.75$ and $86.63 \%$ respectively) (Fig. 1). On the contrary, the item asking to compare doses with equivalent Chest X-ray results in a change of the percentages of correct answers $(96.9 \%$ for US and $80.91 \%$ for MRI).

Only 53.46\% identified that Positron Emission Tomography (PET) scan was the only test among 5 (US, CT, MRI, PET and Mammography) implying a radiation emission by the patient after the exam (radioactive material still inside patient), with a statistically significant difference between radiologists and other doctors $(60.6 \%$ vs $50.4 \%$; $\mathrm{P}$ Fisher's exact test $<0.001$ ).

With the exception of mammography, doctors generally underestimated the radiation doses, and consequently the radiation risk, incurred by the patients undergoing to common radiological investigations. The percentages of correct, underestimated and overestimated answers were summarized in Table 2.

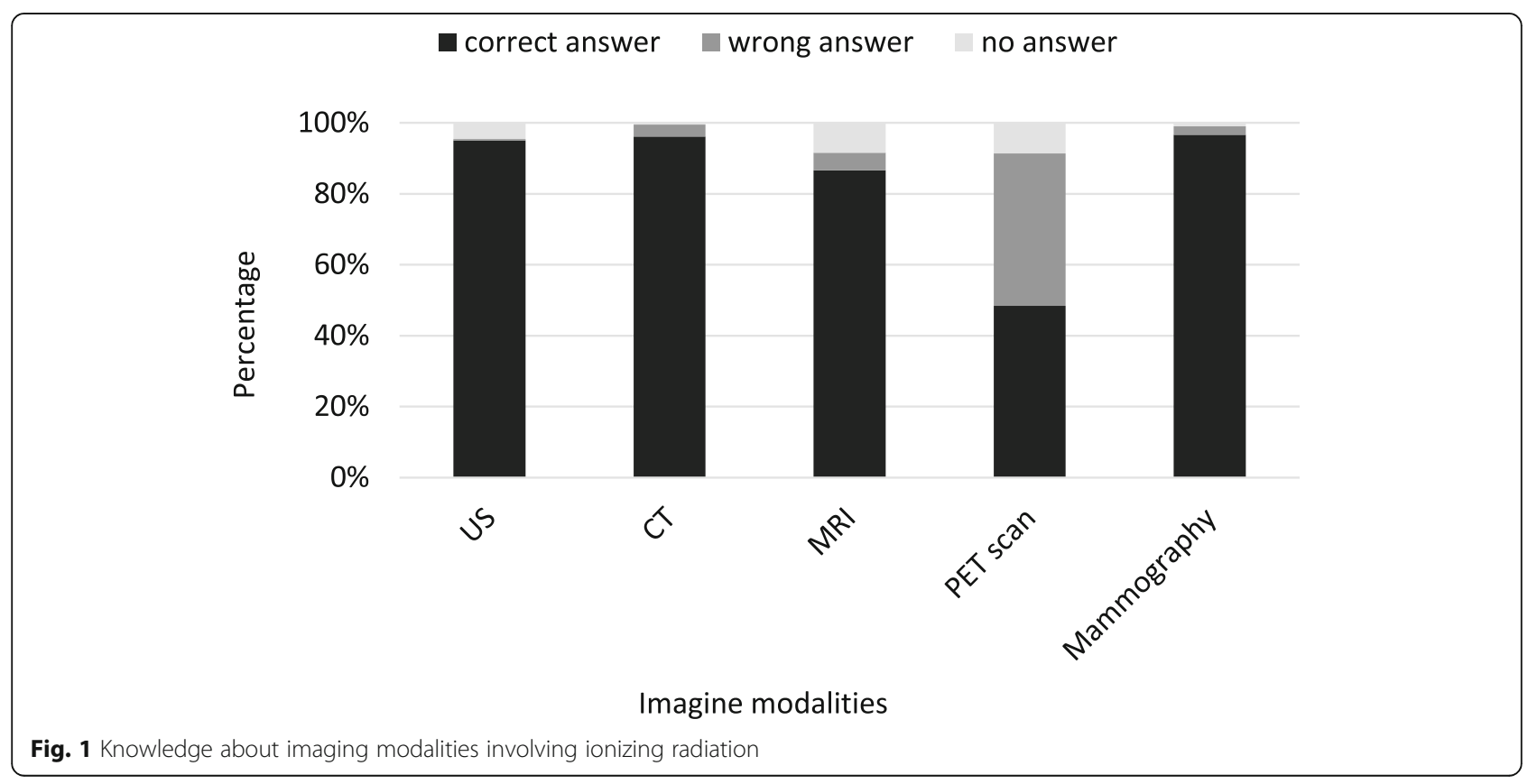


Table 2 Percentage distribution of estimations of doses for radiological modalities

\begin{tabular}{llll}
\hline Modalities & $\begin{array}{l}\text { Underestimated } \\
n(\%)\end{array}$ & $\begin{array}{l}\text { Correct } \\
n(\%)\end{array}$ & $\begin{array}{l}\text { Overestimated } \\
n(\%)\end{array}$ \\
\hline Abdominal CT & $311(74.22)$ & $108(25.78)$ & $0(0)$ \\
Column MR & $0(0)$ & $339(80.91)$ & $80(19.09)$ \\
Abdominal US & $0(0)$ & $406(96.90)$ & $13(3.10)$ \\
Coronary angiography & $362(86.40)$ & $57(13.60)$ & $0(0)$ \\
Mammography & $21(5.01)$ & $350(83.53)$ & $48(11.46)$ \\
\hline
\end{tabular}

$16.71 \%$ of participants properly recognized that almost one radiological procedure involving ionizing radiation increases the malignancy risk. Radiologist correct answers showed a percentage significantly higher than other responders (46.88\% vs $14.71 \% ; p<0.001)$. Only $4.53 \%$ correctly estimated the lifetime risk of inducing a fatal cancer from an abdominal CT (Fig. 2), whereas 19.09\% (80 respondents) believed that there was no risk. This estimation was statistically different between radiologists and other physicians $(12.5 \%$ vs $3.88 \%$, p Fisher $=0.048)$.

Correct choice about possible side effects consequent to more cranial CT was equal to $32.94 \%$ (Fig. 3).

In terms of organ sensitivity to radiation exposure, around the half of respondents (46.06\%) wrongly thought that thyroid was the more sensitive part, without differences between type of medical specialization. $86.26 \%$ of respondents were aware that children were more sensitive than adults and older people to ionizing radiation. Radiologists correctly answer to this topic, with a significant difference with respect to physicians of other specialty $(100 \%$ vs $88.37 \%$; p Fisher $=0.036)$.

Focusing on patient's radiation protection, only 84 responders $(20.05 \%)$ rightly reported that the patient annual dose limit doesn't exist, with a relevant difference between radiologists and other specialists $(43.75 \%$ vs $18.09 \%, p<0.001)$. Most of responders (54.89\%) correctly indicated that $\mathrm{CT}$ accounted for the majority of medical radiation exposure received by the population, but no less than 177 (42.24\%) have chosen conventional radiography. Only $17.66 \%$ correctly chose natural background as the first source of ionizing radiation for the population (Table 3 ).

\section{Discussion}

In our study, the average proportion of correct answers to the questions about ionizing radiation knowledge is $62.29 \%$. The most part of respondents consider very important to be aware about radiation doses of radiological procedures. Less than half of the participants claims to have had previous education on radiation protection and only about $23 \%$ is interested in updating radiation knowledge with specific courses.

Overall, respondents show a knowledge on ionizing radiation higher than those reported in previous similar studies (raging 29\%-40\%) [8-13]. Radiologists appear more knowledgeable than their colleagues, in accordance with evidences from the study performed by Soye et al. [10]. This great knowledge is plausibly in relation with the specific training received during radiology residency.

The frequency of correct answers about US and MRI radiation content is, on average, higher (94.75 and $86.63 \%$, respectively) than the published evidences (ranging $66.1 \%-96 \%$ and $71.4 \%-87.5 \%)$ [9, 10, 13-19]. Surprisingly, the answers to the two questions on the use of ionizing radiation with the same examinations, expressed in different ways give different information. This incongruity highlights the lack of basic knowledge on physic principles involved in the clinical examination.

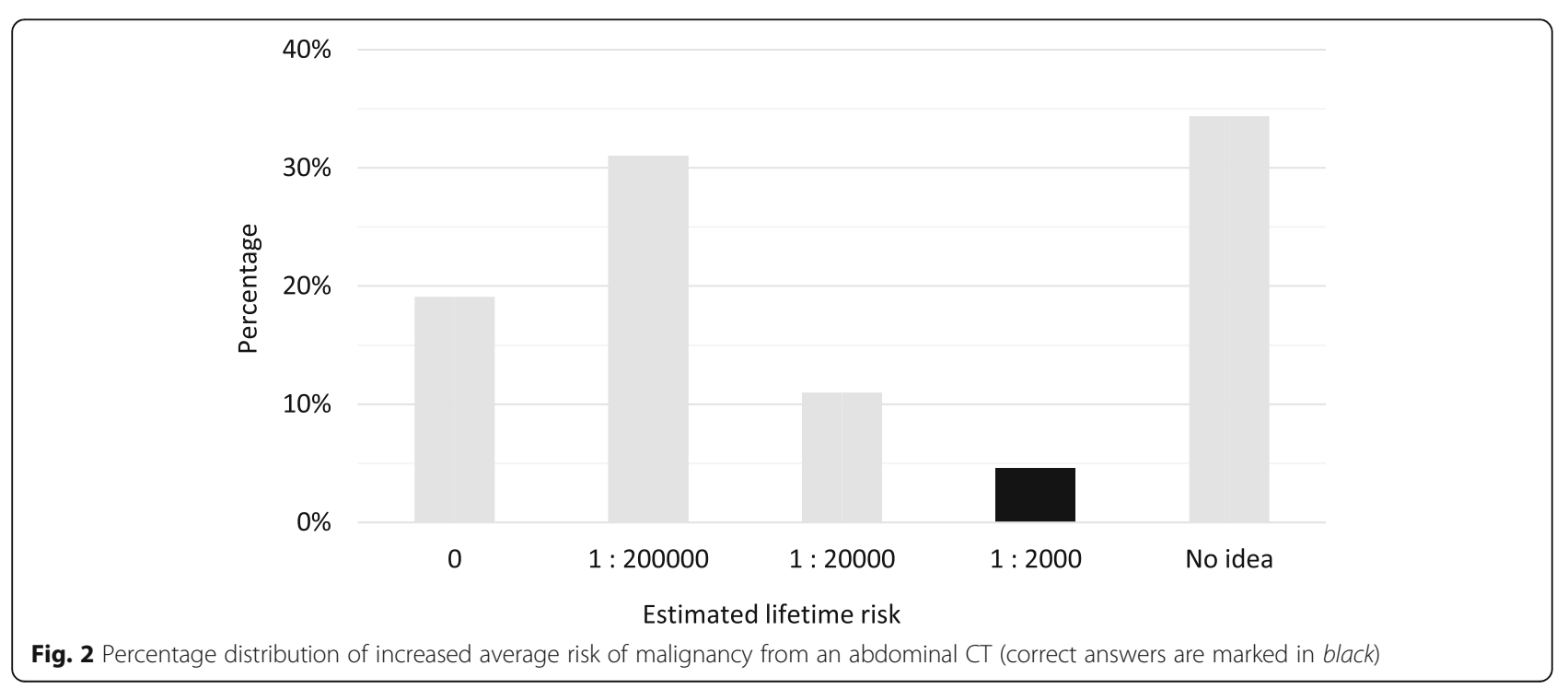




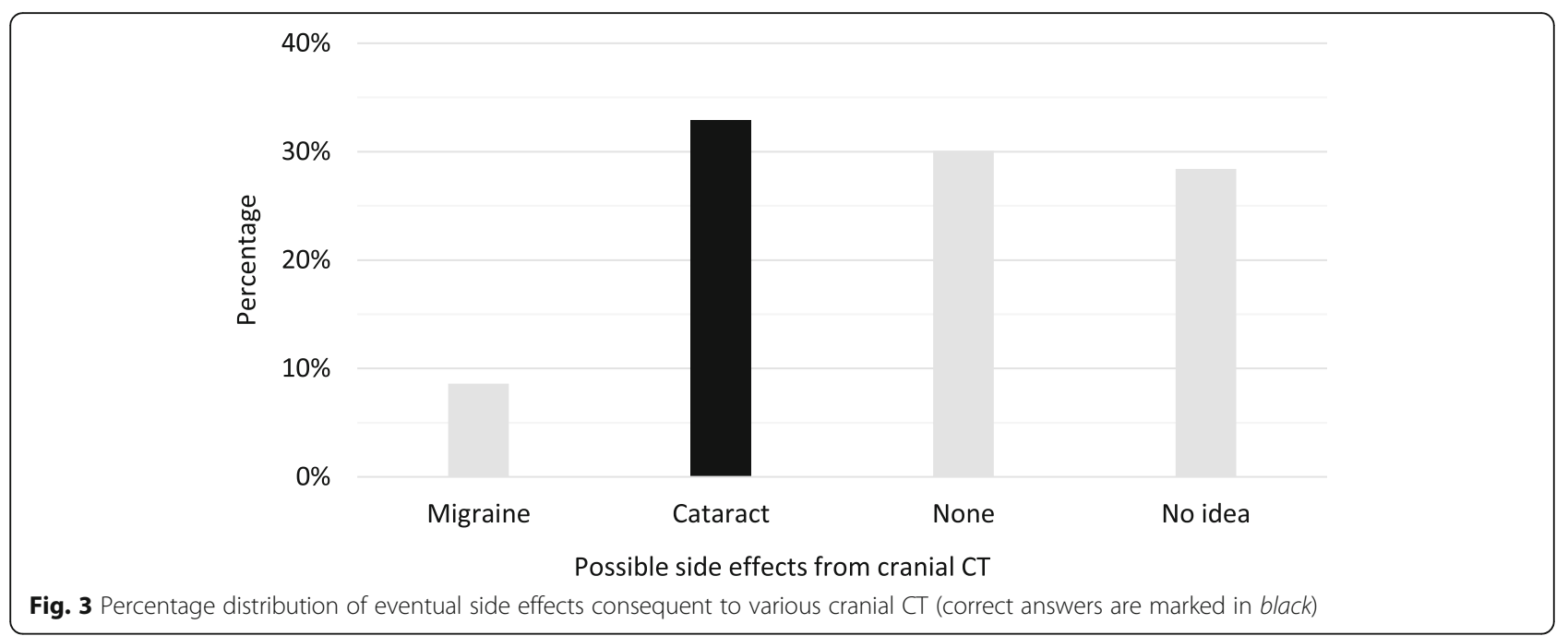

Only $3.34 \%$ of the participants is able to properly evaluate the radiation doses in every single one of the five imaging modalities proposed. In particular, an underestimation of doses for abdominal CT and coronarography (the most risky exams in the list) emerges. These findings are consistent with previous studies $[8,9,13,14,16,18,19]$.

The ratio of the correct answers to the question about the lifetime risk of inducing a fatal cancer from an abdominal CT is in accordance with the evidence from the study of Soylemez et al. [14], performed between Urology residents (3.2\%). In contrast, overseas studies $[10,13,15]$ report correct estimation of the lifetime risk by more responders $(12.5 \%-27.5 \%)$.

The better global performance of our responders compared to those of overseas studies could be due to a more accurate education during medical school, although the most part of doctors declare to have not performed any specific radiation protection training, in accordance with literature.

The present study shows several limits that could influence results. First, the response rate (10.5\%) might introduce a selection bias with a overestimation of several evidences. In addition, the web based methodology could lead to under-representation of physicians with limited access to Internet services. Lastly, the questionnaire used to assess physician's knowledge is not validated nor standardized.

Nevertheless, it is evident that many participants are not enough confident with doses of common imaging tests and with the associated biological effects.

\section{Conclusions}

In conclusion, our findings support the need of raising awareness about risk associated to ionizing radiation procedures. It is imperative to find best modalities and schemes that support a widespread knowledge about radiation protection between physicians and promote the integration of referral guidelines into the clinical practice. In details, we suggest the establishment of a specific course for residents and Continuing Medical Education programs addressed to general practitioners and family pediatricians, who are the first to order imaging tests. The purpose is to minimize unnecessary radiation exposure of the population with improving appropriateness of examinations. It is further to investigate the utility of electronic ad web-based tools as decisional support for doctors in requesting radiological procedures.

Table 3 Percentage distribution of estimations of contribution to collective dose from man-made and natural sources

\begin{tabular}{|c|c|c|c|}
\hline \multirow[b]{2}{*}{ lonizing radiation sources } & \multicolumn{3}{|c|}{$\begin{array}{l}\text { Contribution on collective radiation dose } \\
n(\%)\end{array}$} \\
\hline & 1 & 2 & 3 \\
\hline Medical practices & $139(33.17)$ & $148(35.32)$ & $132(31.51)$ \\
\hline Chernobyl and Fukushima accidents & $203(48.45)$ & $125(29.83)$ & $91(21.72)$ \\
\hline Terrestrial radiation & $221(57.75)$ & $124(29.59)$ & $74(17.66)$ \\
\hline
\end{tabular}

1 = lower contribution, 3 = higher contribution. Correct answer in bold 


\section{Additional file}

Additional file 1: English version of the questionnaire used in the study. (DOCX $17 \mathrm{~kb})$

\section{Abbreviations}

CME: Continuing Medical Education; CT: Computed Tomography; MRI: Magnetic Resonance Imaging; mSv: millisievert; PET: Positron Emission Tomography; US: Ultrasound

\section{Acknowledgements}

The authors would like to thank the Order of Physicians, Surgeons and Dentists of the Pavia District for the support provided.

\section{Funding}

The present work was founded by a budget for research of the Physic Department and the Public Health, Experimental and Forensic Medicine Department of the University of Pavia. The budget support the dispatch of invitation letters and the web platform for questionnaire.

\section{Availability of data and materials}

The dataset is available from the corresponding author upon request.

\section{Authors' contributions}

FC and LR conceived of and planned the study, collected data, performed statistical analysis and participated in the interpretation of results, draft the manuscript. EG conceived of and planned the study, participated in the interpretation of results and made critical revision of the manuscript. PM planned the study, collected data, participated in the interpretation of results, made critical revision of the manuscript. FB planned the study, participated in the interpretation of results and made critical revision of the manuscript. SV planned the study, performed statistical analysis, participated in the interpretation of results and made critical revision of the manuscript. All authors approved the final manuscript.

\section{Competing interests}

The authors confirm that they have no competing interests.

\section{Consent for publication}

Not applicable.

\section{Ethics approval and consent to participate}

The study protocol was approved by the Ethics Committee of the University of Pavia. Informed consent was obtained from each participant simultaneously with completion of the questionnaire.

\section{Publisher's Note}

Springer Nature remains neutral with regard to jurisdictional claims in published maps and institutional affiliations.

\section{Author details}

'Department of Public Health, Experimental and Forensic Medicine, University of Pavia, Via Forlanini 2, 27100 Pavia, Italy. 'Department of Physics, University of Pavia, Via Bassi 6, 27100 Pavia, Italy. ${ }^{3}$ INFN of Pavia, Via Bassi 6, 27100 Pavia, Italy.

Received: 20 October 2015 Accepted: 6 June 2017

Published online: 14 June 2017

\section{References}

1. Agenzia nazionale per la protezione dell'ambiente (APAT). Annuario dei dati ambientali. Estratto. Edizione 2005-2006 (Italian Agency for the Environmental Protection. Annual Report of environmental records. Asbtract. Edition 2005-2006).

2. Berrington de González A, Mahesh M, Kim KP, Bhargavan M, et al. Projected cancer risks from computed tomographic scans performed in the United States in 2007. Arch Intern Med. 2009:169:2071-7.

3. Pérez MR. Referral criteria and clinical decision support: radiological protection aspects for justification. Ann ICRP. 2015:44(1 Suppl):276-87. doi:10.1177/ 0146645314551673
4. ICRP. The 2007 Recommendations of the International Commission on Radiological Protection. ICRP Publication 103. Ann. ICRP 37 (2-4). 2007.

5. Council Directive 2013/59/EURATOM of 5 December 2013 laying down basic safety standards for protection against the dangers arising from exposure to ionising radiation, and repealing Directives 89/618/Euratom, 90/ 641/Euratom, 96/29/Euratom, 97/43/Euratom and 2003/122/Euratom. Official Journal of the European Union 17.1.2014 L 13/1.

6. Hall EJ, Brenner DJ. Cancer risks from diagnostic radiology. Br J Radiol. 2008; 81(965):362-78.

7. Mettler FA, Huda W, Yoshizumi TT, Mahesh M. Effective doses in radiology and diagnostic nuclear medicine: a catalog. Radiology. 2008;248(1):254-63.

8. Bosanquet DC, Green G, Bosanquet AJ, Galland R, et al. Doctors' knowledge of radiation - a two-centre study and historical comparison. Clin Radiol. 2011;66:748-51

9. Zhou GZ, Wong DD, Nguyen LK, et al. Student and intern awareness of ionising radiation exposure from common diagnostic imaging procedures. J Med Imaging Radiat Oncol. 2010;54:17-23.

10. Soye JA, Paterson A. A survey of awareness of radiation dose among health professionals in Northern Ireland. Br J Radiol. 2008;81:725-9.

11. Mola E, Elli P, Orlando T, et al. Valutazione dei bisogni formativi dei medici prescrittori in merito al rischio radiologico. Survey. (A survey on the evaluation of doctor's educational needs regarding radiological risks). Quaderni acp. 2012;19:146-8.

12. Yurt A, Cavusoğlu B, Günay $T$. Evaluation of awareness on radiation protection and knowledge about radiological examinations in healthcare professionals who use ionized radiation at work. Mol Imaging Radionucl Ther. 2014;23:48-53.

13. Keijzers GB, Britton CJ. Doctors' knowledge of patient radiation exposure from diagnostic imaging requested in the emergency department. Med J Aust. 2010;193:450-3.

14. Söylemez H, Sancaktutar AA, Silay MS, et al. Knowledge and attitude of European urology residents about ionizing radiation. Urology. 2013;81:30-5.

15. Divrik Gökçe S, Gökçe E, Coşkun M. Radiology residents' awareness about ionizing radiation doses in imaging studies and their cancer risk during radiological examinations. Korean J Radiol. 2012;13:202-9.

16. Günalp M, Gülünay $B$, Polat $O$, et al. Ionising radiation awareness among resident doctors, interns, and radiographers in a university hospital emergency department. Radiol Med. 2014:119:440-7.

17. Jacob K, Vivian G, Steel JR. X-ray dose training: are we exposed to enough? Clin Radiol. 2004;59:928-34.

18. Arslanoğlu A, Bilgin S, Kubal Z, et al. Doctors' and intern doctors' knowledge about patients' ionizing radiation exposure doses during common radiological examinations. Diagn Interv Radiol. 2007:13:53-5.

19. Singh P, Aggarwal S, Singh Kapoor AM, Kaur R, Kaur A. A prospective study assessing clinicians attitude and knowledge on radiation exposure to patients during radiological investigations. J Nat Sci Biol Med. 2015;6:398-401. doi:10. 4103/0976-9668.160019.

\section{Submit your next manuscript to BioMed Central and we will help you at every step:}

- We accept pre-submission inquiries

- Our selector tool helps you to find the most relevant journal

- We provide round the clock customer support

- Convenient online submission

- Thorough peer review

- Inclusion in PubMed and all major indexing services

- Maximum visibility for your research

Submit your manuscript at www.biomedcentral.com/submit
BioMed Central 